\title{
2019冠状病毒病疫苗接种相关新发及复发 肾小球病研究进展
}

\author{
刘飞,冯春月,毛建华,傅海东 \\ 浙江大学医学院附属儿童医院国家儿童健康与疾病临床医学研究中心国家儿童区域医疗 \\ 中心, 浙江 杭州 310052
}

\begin{abstract}
[摘＼cjkstart要］作为控制疫情的重要手段,2019冠状病毒病(COVID-19)疫苗已经在全 球范围内广泛接种,但疫苗接种引起的新发及复发肾小球病也引起了肾脏病领域 研究者的高度关注。根据全球范围的报道, 信使 RNA 疫苗与灭活疫苗都有可能引 起新发及复发肾小球病; 首剂及第 2 剂接种后都有可能发病; 新发肾小球病以微小 病变为主, 大多对激素敏感; 复发病例亦预后良好, 部分可自行缓解。疫苗引起的体 液、细胞免疫反应可能是导致新发及复发肾小球病的原因。本文总结了 COVID-19 疫苗接种相关的新发及复发肾小球病病例, 并为肾脏病患者接种COVID-19疫苗提供 了建议。
\end{abstract}

[关键词] 2019冠状病毒病; 疫苗; 不良反应; 肾小球病; 新发;复发; 综述 [中图分类号 ］R511;R692［文献标志码］A

\section{New-onset and relapsing glomerular diseases related to COVID-19 vaccination}

LIU Fei, FENG Chunyue, MAO Jianhua, FU Haidong (Children's Hospital, Zhejiang University School of Medicine, National Clinical Research Center for Child Health, National Children's Regional Medical Center, Hangzhou 310052, China) Corresponding author: FU Haidong, E-mail: fhdhz@163.com, https://orcid.org/00000001-9459-3078

\begin{abstract}
[ Abstract ] COVID-19 vaccine, as one of the critical measures to control the pandemic, has been administered in nearly all countries. However, the new-onset and relapsing glomerular diseases associated with COVID-19 vaccination have become a new concern. Both mRNA vaccine and inactivated vaccine may cause new-onset and relapsing glomerular diseases; these diseases would occur after the first dose vaccination or the
\end{abstract}

收稿日期:2021-06-02 接受日期:2021-07-30

基金项目: 浙江省重点研发计划(2019C03028)

第一作者:刘飞,硕士研究生,主要从事儿童肾病综合征、多囊肾的机制研究;E-mail: alexdevin@163.com;https://orcid. org/0000-0001-9098-9155

通信作者:傅海东,主任医师,主要从事儿童肾病综合征的诊疗相关研究;E-mail:fhdhz@163.com; https://orcid.org/00000001-9459-3078 
second dose. New-onset glomerular disease is mainly minimal change glomerulopathy, which is mostly sensitive to steroid, while relapsing cases have good prognosis, and some cases can be spontaneously remitted. The pathogenesis of these vaccine-associated diseases is possibly due to the humoral and cellular immune responses. In this article, we provide a general review of the new-onset and relapsing glomerular diseases related to COVID-19 vaccination, and make suggestions for patients with kidney diseases to receive COVID-19 vaccination.

[ Key words ] Coronavirus disease 2019; Vaccines; Adverse reaction; Glomerulopathy; New-onset; Relapsing; Review

[J Zhejiang Univ (Med Sci), 2021, 50(4): 524-528.]

[缩略语] 2019冠状病毒病(coronavirus disease 2019,COVID-19); 信使RNA(messenger RNA,mRNA); 肾小球微小病变 (minimal change disease, MCD); 美国辉瑞制药 有限公司联合德国生物新技术公司(Pfizer-BioNTech)

COVID-19仍在全球肆虐, 对公众健康构成了 严重威胁。鉴于COVID-19疫情的严重程度, 以及 目前无特效的治疗方法, 接种疫苗成为控制疾病 流行的重要手段。目前约有超百种候选疫苗在世 界范围内研制, 包括灭活疫苗、减毒活疫苗、腺病 毒载体疫苗、重组亚单位疫苗、核酸疫苗 $(m R N A$ 疫 苗和DNA 疫苗) 等 ${ }^{[1]}$ 。截至 2021 年 5 月 25 日, 全球 范围内共接种 1545967545 剂 COVID-19 疫苗 ${ }^{[2]}$ 。

COVID-19疫苗整体保护率为 50\% 95\% ${ }^{[3]}$, 为阻止病毒传播提供了有效的工具。但人们对接 种疫苗后不良反应的担忧一直存在, 特别是部分 接种者在接种疫苗后出现较为严重的不良反应甚 至死亡, 加重了人们对 COVID-19疫苗安全性的担 忧 $^{[4-7]}$ 。近来有文献报道, 接种者在接种COVID-19 疫苗(主要是 mRNA 疫苗及灭活疫苗)后发生新发 肾小球病或原有肾小球病复发 ${ }^{[8-16]}$ 。本文总结了 接种COVID-19疫苗后发生新发肾小球病或原有肾 小球病复发病例的特点, 分析了可能的发病机制, 并对肾脏病患者COVID-19疫苗接种提出了建议。

\section{COVID-19疫苗接种相关新发和复发肾小球 病病例及临床特点}

Lebedev 等 ${ }^{[8]}$ 首先报道了 1 例 50 岁男性患者 接种Pfizer-BioNTech 的 mRNA 疫苗首剂第 3 天出 现腹痛、呕吐、浮肿等临床症状, 实验室检查提示 大量蛋白尿、低蛋白血症及血肌酐升高, 肾穿刺活 体组织检查 (活检)结果提示 MCD伴急性肾小管损
伤, 予醋酸泼尼松 $60 \mathrm{mg} / \mathrm{d}$ 应用约 2 周后尿蛋白转 阴,肌酐恢复正常。D'Agati 等 ${ }^{[9]}$ 随后报道了 1 例 77 岁男性患者接种Pfizer-BioNTech 的 mRNA疫苗 首剂第 7 天出现浮肿症状, 实验室检查提示大量蛋 白尿、低蛋白血症及血肌酐升高, 肾穿刺活检结果 提示 M CD 伴急性肾小管损伤, 予醋酸泼尼松 $60 \mathrm{mg} / \mathrm{d}$ 应用约 $20 \mathrm{~d}$ 复查仍有大量蛋白尿,血肌酤 仍持续偏高。Maas 等 ${ }^{[10]}$ 报道了 1 例 80 岁男性患 者接种 Pfizer-BioNTech 的 mRNA 疫苗首剂第 7 天 出现浮肿症状, 实验室检查符合肾病综合征表现, 肾穿刺活检结果提示 MCD, 予醋酸泼尼松 $80 \mathrm{mg} / \mathrm{d}$ 应用 $10 \mathrm{~d}$ 后尿蛋白/肌酐比值下降至 $0.68 \mathrm{~g} / \mathrm{g}$ 。 Holzworth 等 ${ }^{[11]}$ 报道了 1 例 63 岁女性患者接种美 国Moderna公司 mRNA 疫苗首剂7 d内出现浮肿、 乏力、呼吸困难等临床症状, 辅助检查提示肾病综 合征合并急性肾损伤, 予甲泼尼龙琥珀酸钠 $500 \mathrm{mg}$ 冲击 $3 \mathrm{~d}$ 后改为醋酸泼尼松 $1 \mathrm{mg} \cdot \mathrm{kg}^{-1} \cdot \mathrm{d}^{-1}$ 口服, 结局不详。这 4 例新发肾病患者均接种 mRNA 疫苗,平均年龄为 67.5 岁; 起病时间为首剂 疫苗接种后第3 7天, 3 例合并肾功能损害, 3 例肾 穿刺活检结果提示 MCD ; 经激素治疗后, 2 例患者 临床症状迅速缓解, 1 例结局不详, 1 例短期疗效不 佳, 需长期随访观察。

Aydin 等 ${ }^{[12]}$ 报道了1例66岁原发性膜性肾病 女性患者接种北京科兴生物制品有限公司灭活 疫苗首剂 2 周后出现浮肿症状, 尿蛋白/肌酤 $9.42 \mathrm{~g} / \mathrm{g}$, 血肌酐 $245.8 \mu \mathrm{mol} / \mathrm{L}$, 后续治疗及转归 
不详。Kervella 等 ${ }^{[13]}$ 报道了 1 例 34 岁激素依赖性 肾病综合征(病理表现为 MCD) 女性患者接种 Pfizer-BioNTech 的 mRNA 疫苗首剂第 10 天尿 蛋白 $/$ 肌䣶 $2.4 \mathrm{~g} / \mathrm{g}$, 第 2 剂数天后尿蛋白/肌䣶 $3.0 \mathrm{~g} / \mathrm{g}$, 将醋酸泼尼松加量至 $1 \mathrm{mg} \cdot \mathrm{kg}^{-1} \cdot \mathrm{d}^{-1}$ 后 完全缓解。Komaba 等 ${ }^{[14]}$ 报道了 1 例 65 岁肾病 综合征(病理表现为 MCD) 男性患者接种 PfizerBioNTech 的 mRNA 疫苗首剂第 10 天出现泡沫尿, 尿蛋白/肌䣶 $11.48 \mathrm{~g} / \mathrm{g}$, 白蛋白 $28 \mathrm{~g} / \mathrm{L}$; 予醋酸泼 尼松 $20 \mathrm{mg} / \mathrm{d}$ 、环孢素 $100 \mathrm{mg} / \mathrm{d}$ 治疗 2 周后尿蛋白 转阴。Rahim 等 ${ }^{[15]}$ 报道了 1 例 52 岁 IgA 肾病女性 患者接种Pfizer-BioNTech 的 mRNA疫苗第 2 剂 $24 \mathrm{~h}$ 内出现肉眼血尿, 尿蛋白/肌酔 $4.2 \mathrm{~g} / \mathrm{g}$, 继续原血 管紧张素转化酶抑制剂治疗 1 周内自行缓解。 Negrea等 ${ }^{[16]}$ 报道了 2 例 38 岁 IgA 肾病女性患者接种 Pfizer-BioNTech 的mRNA疫苗第2剂 $24 \mathrm{~h}$ 内出现肉 眼血尿, 其中 1 例患者出现尿蛋白定量增加, 继续原 肾素-血管紧张素-醛固酮系统抑制剂治疗后, 2 例 患者皆在 $3 \mathrm{~d}$ 内自行缓解。此 6 例肾病复发患者中, 5 例接种 $m R N A$ 疫苗, 1 例接种灭活疫苗; 复发时间 多出现在首剂后 1 2周或第 2 剂接种后 $24 \mathrm{~h}$ 内; 5 例 mRNA 疫苗接种病例经激素及免疫抑制剂调整后 病情缓解, 1例灭活疫苗接种病例预后不详。

综上可见, mRNA 疫苗和灭活疫苗均可能引 起新发及复发肾小球病; 发病时间可能在首剂接 种后, 也可能在第2 剂接种后; 新发肾小球病多合 并急性肾损伤, 病理类型以 MCD 为主; 新发病例多 对激素敏感, 复发病例经药物调整治疗后预后良 好,部分可自行缓解。

接种者在疫苗接种后出现新发及复发肾小球 病表现, 首要问题是疾病与疫苗接种是巧合还是 因果关系。临床出现此类病例, 判断的依据主要 是发病的时间点及排他性的诊断。对于原发病 例, 如条件允许可考虑行肾穿刺活检, 如表现为 $\mathrm{MCD}$, 可予激素治疗, 多可获缓解; 对于复发病例, 通过调整激素及免疫抑制剂的剂量等, 多数亦可 快速缓解。

\section{COVID-19疫苗接种相关新发及复发肾小球 病的发病机制}

$\mathrm{mRNA}$ 疫苗引人编码疾病特异性抗原的 mRNA, 利用宿主细胞的蛋白质合成机制产生抗 原, 从而触发免疫应答。mRNA 疫苗接种后会触
发机体的体液免疫和细胞免疫,一方面通过被 $\mathrm{CD} 8^{+} \mathrm{T}$ 细胞识别从而诱导细胞介导的免疫反应, 另一方面通过被 $\mathrm{CD} 4^{+} \mathrm{T}$ 细胞识别促进 $\mathrm{B}$ 细胞产生 抗原特异性抗体, 进一步促进细胞因子及炎症介 质的释放 ${ }^{[17]}$ 。前期临床试验也观察到 PfizerBioNTech 的 mRNA 疫苗会引起强烈的 $\mathrm{CD} 4^{+}$和 $\mathrm{CD}^{+} \mathrm{T}$ 细胞反应和抗体反应及细胞因子释放 ${ }^{[18]}$ 。 肾小球病与 $\mathrm{T}$ 细胞、 $\mathrm{B}$ 细胞介导的足细胞损伤密切 相关, 因此 mRNA疫苗介导的体液、细胞免疫反应 可能是引起足细胞损伤导致新发及复发肾小球病 的原因 ${ }^{[19]}$ 。COVID-19 灭活疫苗相关肾小球病与 流感等其他灭活疫苗引起的 MCD 有相似之处, 也 与疫苗引起的免疫反应有关 ${ }^{[20-21]}$ : 灭活疫苗直接 引入抗原蛋白, 从而刺激宿主免疫应答, 引起 $\mathrm{T}$ 细 胞、 $\mathrm{B}$ 细胞的反应, 最终导致足细胞损伤。既往也 有疫苗相关血管炎复发的报道, 提示可能与迟发 变态反应也有关 ${ }^{[22-23]}$ 。此外, 不仅疫苗本身, 佐剂 也可能触发机体的免疫反应 ${ }^{[24]}$ 。

mRNA疫苗模拟病毒触发人体对感染的免疫 应答,对比病毒感染后的自然适应性反应,一般在 疫苗接种后早期 (2 4 d ) 就可观察到 $\mathrm{T}$ 细胞激活, 细胞内的免疫反应多在感染后 1 周发生 ${ }^{[25]}$ 。故疫 苗接种后早期及 1 周左右可能是新发及复发肾小 球病的高发时间,这与已有报道大致吻合。从病 理学机制上看,第 2 剂疫苗的免疫应答更强烈,但 已有报道中仅 3 例是发生在第 2 剂疫苗接种后, 后 续需要更深人研究两者之间的联系。接种者的基 础情况、激素及免疫抑制剂的用量可能对新发及 复发肾小球病均有影响 ${ }^{[26]}$ 。

\section{3 免疫相关肾脏病患者 COVID-19 疫苗接种 建议}

免疫性疾病患者及应用免疫抑制剂患者在 COVID-19疫苗前期临床试验中多被排除在外，但 考虑到疫苗诱导抵抗重症 COVID-19的免疫潜力 超过了可能潜在的风险，欧洲肾脏协会一欧洲透析 和移植协会免疫肾脏病工作组建议对所有免疫介 导的肾脏病患者进行 COVID-19疫苗接种(已知对 疫苗成分过敏者除外 $)^{[27-28]}$ 。目前, mRNA 疫苗、 灭活疫苗、腺病毒载体疫苗均表现出良好的免疫 原性及保护力, 参照国内特殊人群 (慢性肝病、结 核病和风湿免疫病患者)COVID-19疫苗接种专家 意见 ${ }^{[29]}$ 及欧洲肾脏协会的推荐, 对于免疫相关肾 
脏病患者接种 COVID-19 疫苗有以下几点建 议: (1) 建议首选接种灭活疫苗, 慎重考虑接种 $\mathrm{mRNA}$ 疫苗、腺病毒载体疫苗、重组亚单位疫 苗; (2)疫苗接种前应咨询专科医生, 接种时糖皮质 激素应维持在泼尼松等量剂量低于 $20 \mathrm{mg} / \mathrm{d}$, 利妥 昔单抗使用后至少间隔 6 个月以上, 其他如甲氨蝶 呤、JAK 抑制剂、环磷酰胺建议在每一剂疫苗接种 后暂停使用 1 周; (3)接种后须密切观察基础疾病的 病情, 监测疾病活动度, 应用多种免疫抑制剂、前 期有 COVID-19相关病毒感染史、高龄者须重点关 注; (4)接种后应继续遵循手卫生、社交距离等防疫 要求。

总的来说,COVID-19疫苗仍是终止 COVID-19 流行的最终希望。根据前期的临床试验结果, COVID-19疫苗整体保护率高、不良反应少,但仍 不能忽视可能的严重不良反应及特殊人群的接种 反应。在肾脏病领域, 后期扩大范围接种时须密 切关注有无疾病新发及复发, 监测肾脏病患者接 种后免疫状态的变化, 警惕严重不良反应的发 生。采取必要的个体化接种策略(包括合理的接 种时机)有助于促进肾脏病患者COVID-19疫苗接 种获益。未来希望总结更多病例, 探索有肾脏基 础疾病患者COVID-19疫苗的个体化接种策略, 以 及 COVID-19疫苗接种与新发和复发肾小球病的 深层次关系。

\section{利益冲突 所有作者均声明不存在利益冲突}

\section{参考文献}

[1] LI Y, TENCHOV R, SMOOT J, et al. A comprehensive review of the global efforts on COVID-19 vaccine development [J]. ACS Cent Sci, 2021, 7(4): 512-533.

[2] World Health Organization. Draft landscape and tracker of COVID-19 candidate vaccines [EB/OL]. [2021-0525]. https://www.who.int/publications/m/item/draftlandscape-of-covid-19-candidate-vaccines.

[3] CARNEIRO D C, SOUSA J D, MONTEIRO-CUNHA J P. The COVID-19 vaccine development: a pandemic paradigm[J]. Virus Res, 2021, 301: 198454.

[4] SCHULTZ N H, SØRVOLL I H, MICHELSEN A E, et al. Thrombosis and thrombocytopenia after ChAdOx1 nCoV-19 vaccination [J]. N Engl J Med, 2021, 384(22): 2124-2130

[5] BOZKURT B, KAMAT I, HOTEZ P J. Myocarditis with COVID-19 mRNA vaccines[J]. Circulation, 2021, 144(6): 471-484.
[6] CASTELLS M C, PHILLIPS E J. Maintaining safety with SARS-CoV-2 vaccines[J]. N Engl J Med, 2021, 384(7): 643-649.

[7] FORMEISTER E J, CHIEN W, AGRAWAL Y, et al. Preliminary analysis of association between COVID-19 vaccination and sudden hearing loss using US centers for disease control and prevention vaccine adverse events reporting system data $[\mathrm{J}]$. JAMA Otolaryngol Head Neck Surg, 2021, 147(7): 674-676.

[8] LEBEDEV L, SAPOJNIKOV M, WECHSLER A, et al. Minimal change disease following the Pfizer-BioNTech COVID-19 vaccine[J]. Am J Kidney Dis, 2021, 78(1): 142-145.

[9] D'AGATI V D, KUDOSE S, BOMBACK A S, et al. Minimal change disease and acute kidney injury following the Pfizer-BioNTech COVID-19 vaccine[J]. Kidney Int, 2021, 100(2): 461-463.

[10] MAAS R J, GIANOTTEN S, VAN DER MEIJDEN W A G. An additional case of minimal change disease following the Pfizer-BioNTech COVID-19 vaccine $[\mathrm{J}]$. Am J Kidney Dis, 2021, 78(2): 312.

[11] HOLZWORTH A, COUCHOT P, CRUZ-KNIGHT W, et al. Minimal change disease following the moderna mRNA-1273 SARS-CoV-2 vaccine $[J]$. Kidney Int, 2021, 100(2): 463-464.

[12] AYDIN M F, YILDIZ A, ORUÇ A, et al. Relapse of primary membranous nephropathy after inactivated SARS-CoV-2 virus vaccination[J]. Kidney Int, 2021, 100(2): 464-465.

[13 ] KERVELLA D, JACQUEMONT L, CHAPELET-DEBOUT A, et al. Minimal change disease relapse following SARS-CoV-2 mRNA vaccine[J]. Kidney Int, 2021, 100(2): 457-458.

[14] KOMABA H, WADA T, FUKAGAWA M. Relapse of minimal change disease following the Pfizer-BioNTech COVID-19 vaccine[J]. Am J Kidney Dis, 2021, 78(3): 469-470.

[15 ] RAHIM S E G, LIN J T, WANG J C. A case of gross hematuria and $\operatorname{IgA}$ nephropathy flare-up following SARS-CoV-2 vaccination $[J]$. Kidney Int, 2021, 100(1): 238.

[16] NEGREA L, ROVIN B H. Gross hematuria following vaccination for severe acute respiratory syndrome coronavirus 2 in 2 patients with IgA nephropathy $[\mathrm{J}]$. Kidney Int, 2021, 99(6): 1487.

[17] XU S, YANG K, LI R, et al. mRNA vaccine eramechanisms, drug platform and clinical prospection $[\mathrm{J}]$. Int J Mol Sci, 2020, 21(18): 6582.

[18] SAHIN U, MUIK A, DERHOVANESSIAN E, et al. COVID-19 vaccine BNT162b1 elicits human antibody and TH1 T cell responses $[\mathrm{J}]$. Nature, 2020, 586(7830): 594-599. 
[19] VIVARELLI M, MASSELLA L, RUGGIERO B, et al. Minimal change disease $[\mathrm{J}]$. Clin J Am Soc Nephrol, 2017, 12(2): 332-345.

[20] KIELSTEIN J T, TERMÜHLEN L, SOHN J, et al. Minimal change nephrotic syndrome in a 65 -year-old patient following influenza vaccination $[\mathrm{J}]$. Clin Nephrol, 2000, 54(3): 246-248.

[21] GUTIERREZ S, DOTTO B, PETITI J P, et al. Minimal change disease following influenza vaccination and acute renal failure: just a coincidence? $[\mathrm{J}]$. Nefrologia, 2012, 32(3): 414-415.

[22] LAMBERT E M, LIEBLING A, GLUSAC E, et al. Henoch-schonlein purpura following a meningococcal vaccine $[\mathrm{J} / \mathrm{OL}]$. Pediatrics, 2003, 112(6): e491-e494.

[23] MCNALLY A, MCGREGOR D, SEARLE M, et al. Henoch-Schonlein purpura in a renal transplant recipient with prior IgA nephropathy following influenza vaccination $[\mathrm{J}]$. Clin Kidney J, 2013, 6(3): 313-315.

[24] SEGAL Y, SHOENFELD Y. Vaccine-induced autoimmunity: the role of molecular mimicry and immune crossreaction $[\mathrm{J}]$. Cell Mol Immunol, 2018, 15(6): 586-594.

[25] SETTE A, CROTTY S. Adaptive immunity to SARSCoV-2 and COVID-19[J]. Cell, 2021, 184(4): 861-880.
[26] SOY M, KESER G, ATAGUNDUZ P, et al. A practical approach for vaccinations including COVID-19 in autoimmune/autoinflammatory rheumatic diseases: a non-systematic review $[\mathbf{J}]$. Clin Rheumatol, 2021, 40(9): 3533-3545.

[27 ] DAL-RÉ R, BEKKER L G, GLUUD C, et al. Ongoing and future COVID-19 vaccine clinical trials: challenges and opportunities [J]. Lancet Infect Dis, 2021.

[28] KRONBICHLER A, ANDERS H J, FERNANDEZJUÁREZ G M, et al. Recommendations for the use of COVID-19 vaccines in patients with immune-mediated kidney diseases $[\mathrm{J}]$. Nephrol Dial Transplant, 2021, 36(7): 1160-1168.

[29］中华医学会感染病学分会, 中华医学会风湿病学分 会. 特殊人群(慢性肝病、结核病和风湿免疫病患者) 新型冠状病毒疫苗接种专家建议 $[\mathrm{J}]$. 中华传染病 杂志, 2021, 39(7): 398-403.

Chinese Society of Infectious Diseases, Chinese Society of Rheumatology. Expert recommendation on severe acute respiratory syndrome coronavirus 2 vaccination in patients with chronic liver diseases, tuberculosis or rheumatoid diseases $[\mathrm{J}]$. Chinese Journal of Infectious Diseases, 2021, 39(7): 398403. (in Chinese)

[本文编辑 沈 敏 余 方]

\section{杨巍教授和罗建红教授团队揭示N-甲基-D-门冬氨酸受体 GluN2B磷酸化修饰介导抑有症新机制}

2021年8月24日,浙江大学医学院杨巍教授和罗建红教授团队在《细胞报告》(Cell Reports)在线发表了研究论文 “Disrupting phosphorylation of Tyr-1070 at GluN2B selectively produces resilience to depression like behaviors” (https:// pubmed. ncbi. nlm. nih. gov/34433031/)。文章揭示了内侧前额叶皮层N-甲基-D-门冬氨酸受体(NMDAR)GluN2B亚基酪 氨酸1070位点磷酸化选择性调控介导抗抑有样行为的新机制。

研究人员运用CRISPR-Cas9技术构建了GluN2B Y1070F的遗传突变小鼠,采用系列行为学检测意外发现突变小鼠在 基础水平及 $14 \mathrm{~d}$ 慢性束缚应激暴露后均表现出显著抗抑郁样行为,而认知功能、焦虑、恐惧记忆、社交能力和探索等行为 却无变化, 提示该突变选择性影响了小鼠抑郁相关行为。进一步研究发现,突变小鼠前额叶皮层的GluN2B酪氨酸1472 位点磷酸化水平降低,表现出了脑区选择性的调控作用。脑片电生理检测结果证明突变小鼠前额叶皮层第5层雉体神经 元的突触NMDAR功能正常, 但突触外GluN2B亚型NMDAR功能显著下调, 表明酪氨酸1070位点选择性调控非突触 NMDAR的细胞膜定位。相反,海马雉体神经元的突触内外NMDAR均未变化,这进一步明确该机制具有脑区选择性。随 后,通过组合脑片电生理、生化检测和高尔基染色等手段发现,突变小鼠前额叶皮层的mTORC1活性显著增强,导致神经 元兴奋性突触数量增加, 从而产生了抗抑郁样行为。

师小芳博士为论文第一作者。研究得到国家自然科学基金和浙江省自然科学基金等资助。 